Espacio, Tiempo y Forma, Serie VII, H. ${ }^{a}$ del Arte, t. 16, 2003, págs. 19.9-221

\title{
Centrales telefónicas norteamericanas en los años 20
}

\author{
Francisco Javier García Algarra \\ Doctorado Historia del Arte. UNED
}

\section{RESUMEN}

Las centrales telefónicas son edificios muy especiales debido a la variedad de propósitos que sirven. Albergan los equipos industriales de la red de telecomunicaciones y también fueron diseñadas para mostrar la riqueza de las empresas propietarias. Durante los años 20, AT\& T encargó muchas centrales nuevas para atender una demanda explosiva en EE UU. El exitoso modelo americano fue imitado por las principales empresas telefónicas del mundo y lo adoptó la Compañía Telefónica Nacional de España, fundada en 1924 como filial de ITT.

\begin{abstract}
Telephone Central Offices are buildings very special, due to a mixture of purposes that they serve. They host the industrial equipment of the telecommunications network but they were also designed to show the wealth of the owner companies. During the roaring 20's, AT\&T commanded a lot of new Central Offices to deal with a explosive telephone demand in the USA. The successful American model was followed by the main telephone companies of the world, and it was adopted by the Compañia Telefónica Nacional de España, founded as an ITT subsidiary in 1924.
\end{abstract}

\section{INTRODUCCIÓN}

Las centrales telefónicas son un tipo de edificación concebido para albergar los equipos que permiten prestar un servicio imprescindible para la sociedad contemporánea, pero a diferencia de otras redes públicas de transporte o distribución, la telefonía no ha sido hasta el momento objeto preferente de estudio de la arqueología industrial.

El carácter industrial de esta infraestructura no es fácilmente perceptible desde el exterior de las centrales. Gran parte de las instalaciones fabriles de las pri- 
meras décadas del siglo xx han desaparecido por obsolescencia. La catalogación y protección de este patrimonio han favorecido la investigación y el aprecio de sus valores estéticos. Sin embargo, la mayoría de las centrales continúan prestando el mismo servicio para el que fueron originalmente construidas, y no es previsible que dejen de hacerlo a corto plazo. Esto puede resultar sorprendente teniendo en cuenta que pueden localizarse en zonas privilegiadas de las grandes ciudades ${ }^{1}$ pero una razón de naturaleza económica ha contribuido a esta longevidad. La distancia máxima del par de cables que unen al abonado con la central es de unos cinco kilómetros, debido a una limitación técnica. Por esta causa las centrales se encuentran dispersas por toda la ciudad, en medio de edificios de todo tipo. Pese a que la tecnología ha evolucionado de forma espectacular, el coste en tiempo, dinero e interrupción del servicio que supondría cambiar todo el cableado que une los domicilios de los usuarios con su central para trasladar el edificio es inabordable, un problema de magnitud comparable al de modificar el ancho de la red ferroviaria. Eso hace que sean muy escasas las que hayan cambiado de uso y que no se encuentren apenas ejemplos de edificios en ruina.

Otra peculiaridad que se deriva del hecho anterior es el crecimiento del edificio original en anchura y altura por sucesivas adiciones. Las ampliaciones se van acometiendo a medida que la demanda aumenta sin que, por regla general, se respete el estilo del primer arquitecto. Las centrales producen así, en unas pocas décadas, un fenómeno general en aquellos edificios de uso continuado durante mucho tiempo, como las catedrales.

El objetivo de este trabajo ha sido estudiar la arquitectura de las grandes centrales telefónicas de Estados Unidos en los años veinte, como paso previo para una futura investigación sobre el patrimonio inmobiliario de Telefónica de España. El desarrollo de la telefonía en América del Norte tuvo lugar con gran adelanto sobre Europa en general y España en particular. Entre el final de la Primera Guerra Mundial y el crack de 1929, la expansión económica favoreció la extensión del servicio ${ }^{2}$. La

1 Un caso muy llamativo es el del edificio Barclay-Vesey situado a escasos metros de Wall Street. Su exterior e instalaciones sufrieron graves daños por el derrumbe de las Torres Gemelas y el edificio 7 del World Trade Center el 11 de septiembre de 2001. La compañía Verizon (heredera directa de la New York Telephone Company) hizo un esfuerzo extraordinario por restaurar el servicio que presta a 300.000 líneas de teléfono y 3,6 millones de circuitos de datos durante el mes que siguió a la tragedia. Ni siquiera en esas circunstancias se planteó el traslado. El 15 de octubre, el vicepresidente de Verizon, Lawrence Babbio, en declaraciones al New York Times, afirmó: «It would take Verizon five years to build alternate pathways for all the telephone lines that wind their way into and out the New York Telephone Building. And Verizon has no plans to do so".

2 Romero Frías, R.: “Colección histórico-tecnológica de Telefónica». Telefónica de España, Madrid, 1992, pág. 22: «A pesar de que en 1900 existían ya todos los sistemas de comunicación que constituyen la Telecomunicación, si bien es verdad que en estado muy incipiente, no es hasta entrada la década de los años veinte cuando se aplican eficazmente para prestar servicios útiles a la sociedad". 

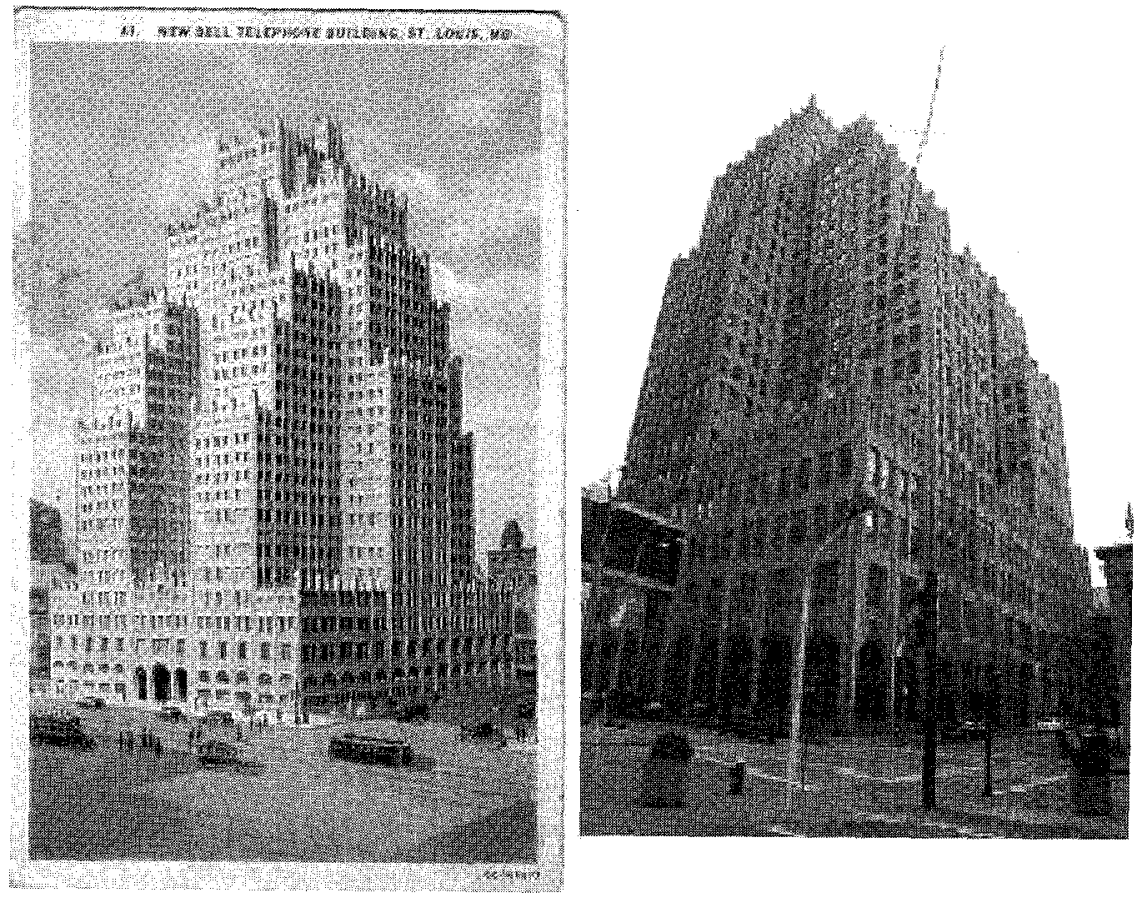

Fig. 1. La central de St. Louis, edificada en 1925, en una postal de época y en la actualidad. Fuente: Mound City of the Mississipi.

todopoderosa «American Telephone \& Telegraph» llevó a cabo amplios programas de inversión para atender el incremento de la demanda. La forma de trabajar de AT\&T se impuso como modelo para el resto de compañías, como la ITT, hecho fundamental para poder comprender el desarrollo inmobiliario de su filial española en sus primeros años de existencia. Las características de este sistema americano que fue adaptado en España con pequeñas variaciones son las siguientes:

- Existencia de una oficina central, dependiente del departamento de ingeniería que establece las normas técnicas y unas líneas generales a las que deben atenerse los proyectos arquitectónicos. La construcción de centrales se concibe como una tarea más del proceso conocido como creación de planta.

- Encargo de la redacción de proyectos y ejecución de las obras a un reducido grupo de estudios de arquitectura que se especializan en este tipo de edificación y consiguen unos plazos adecuados al resto de la creación de 
planta $^{3}$. El funcionamiento de estos estudios tenía poco que ver con sus equivalentes europeos de la época pues se trataba de oficinas de proyectos con decenas o incluso centenares de empleados con una estricta división del trabajo.

- Dentro de las limitaciones que imponen los requisitos técnicos, los arquitectos disponían de libertad en la concepción de las obras. Aunque las centrales son edificios de carácter utilitario se decoraron de forma profusa con el objetivo de mostrar a los usuarios y accionistas el poder de la compañía propietaria. La utilización de motivos propios de la arquitectura de cada ciudad junto con el uso de elementos historicistas, fantásticos o art-déco contribuyeron a conferir personalidad a unos edificios condenados a repetirse.

Es importante no perder de vista esta originalidad. En cada época los arquitectos deben dar respuesta a problemas novedosos y la que encontraron los arquitectos norteamericanos en los años 20 marcó el desarrollo de toda la construcción de centrales. Algunas de ellos, son exponentes de la mejor arquitectura de su época. Sin duda, el edificio Barclay-Vesey de Ralph Thomas Walker para la New York Telephone Company es el modelo que influyó en todos los posteriores. Fue además uno de los edificios más admirados de la década. Le Corbusier utilizó su fotografía como portada en la edición inglesa de "Hacia una nueva arquitectura» de 1927, en la que afirma que «se puede encontrar la arquitectura en el teléfono y en el Partenón».

\section{LA AUTOMATIZACIÓN}

La esencia del servicio telefónico es poner en contacto a dos abonados cualesquiera. Para conseguirlo es necesario que alguien o algo, conecte los dos cables del abonado que llama con los de la persona llamada. Este proceso, llamado conmutación, se lleva a cabo desde la invención del teléfono en las centrales. Al principio, se hacía de forma manual. La operadora preguntaba con quien se quería hablar y mediante una serie de clavijas establecía la conexión.

Las centrales de finales del siglo XIX y principios del XX eran edificios no muy grandes que alojaban los primitivos conmutadores manuales $y$, por supuesto, a las operadoras. El teléfono era un servicio caro, utilizado sólo por las clases más pudientes y, por limitaciones técnicas, casi todas las llamadas eran urbanas. Hasta

${ }^{3}$ ARMS, M.: «The architecture of Ralph Thomas Walker: 1919-1930». Tesis doctoral, Universidad de Rutgers, 1995. pág. 94: “Each of the American Telephone and Telegraph Company's twenty-four subsidiaries maintained an association with an architect who was responsible for ensuring that the design of new telephone buildings was in keeping with architectural trends and with the architectural climate of the regions for which they were designedn. 


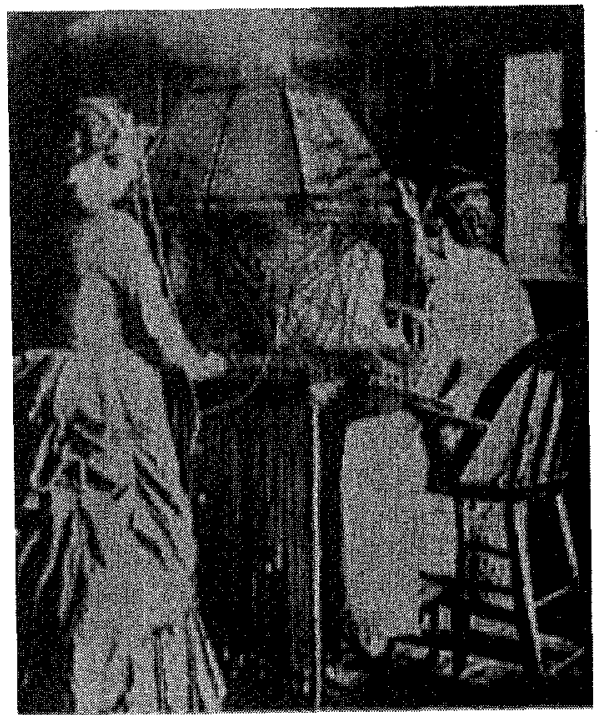

Fig. 2. Operadoras trabajando en un conmutador manual arcaico en 1882. Fuente: Bell System Memorial.

1919, la AT\&T y todas sus compañías filiales mantuvieron el sistema manual, aunque ya desde finales del siglo XIX se había desarrollado un sistema de conmutación completamente automático. Al término de la Gran Guerra, el negocio cambió de forma radical. En 1915, los ingenieros de la compañía consiguieron diseñar un sistema que permitía establecer llamadas de costa a costa. Desde ese momento, la empresa se planteó como objetivo estratégico el «servicio universal». La expansión económica que siguió a la guerra impulsó el negocio y el teléfono pasó a ser un bien de consumo masivo.

A partir de un umbral de tráfico las operadoras humanas son más caras que los equipos, pese a que sus salarios siempre fueron reducidos, pero lo que hizo cambiar por completo el modo de pensar de la dirección fue una huelga de operadoras en el año 1920 que mostró la vulnerabilidad de la compañía ante el factor humano. Las condiciones para la construcción en serie de centrales con equipos automáticos estaban dadas.

La instalación de estos sistemas hizo cambiar los criterios de diseño arquitectónico. Se trata de equipos muy pesados, una estructura de central puede requerir forjados capaces de soportar entre 850 y $1000 \mathrm{~kg} / \mathrm{m}^{2}$, cifra muy superior a la de una oficina en la que es del orden de $250 \mathrm{~kg} / \mathrm{m}^{2}$. Como contrapartida no necesitan unas condiciones de ventilación e iluminación como las de las salas de operadoras. 


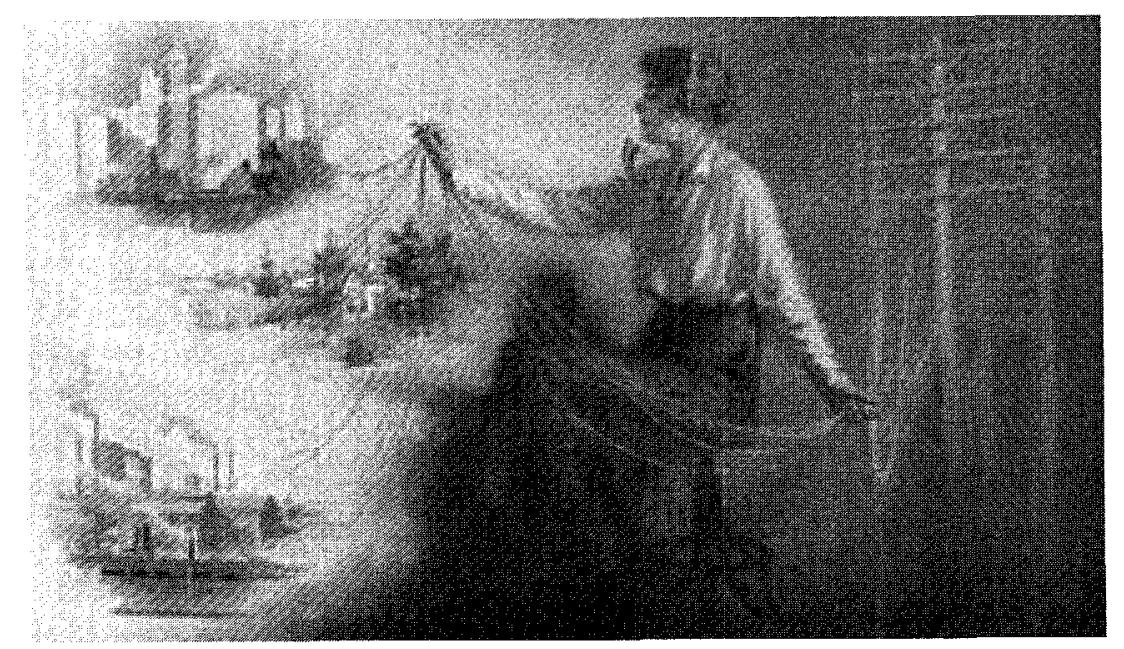

Fig. 3. Imagen de un anuncio de AT\&T de 1929. La operadora es una alegoría del servicio universal que une a todo el país. Fuente: Bell System Memorial.

Estas circunstancias impusieron una característica distribución en altura. Las baterías y los electrogeneradores se instalaban, por regla general, en los sótanos. En la planta baja se sitúa el repartidor principal del edificio y los sistemas de transmisión y conmutación en los pisos superiores. Sobre estos, se acondicionaban las salas de operadoras y las oficinas, y en los cuarteles generales se reservaba la planta superior a despachos de los directivos.

Otra singularidad de los edificios telefónicos es la altura de sus plantas, determinada por el tamaño de los equipos. Esta dimensión colosal, al contrario de lo que pueda parecer, no se diseñó así con la intención de crear vestíbulos para impresionar a los visitantes. Estos fueron la consecuencia y no la causa de una necesidad técnica.

En las principales ciudades se concentraban en la oficina principal equipos automáticos, operadoras, servicios administrativos y atención al público. Esta conjunción de necesidades diversas da origen a otra de las características de la arquitectura de centrales. Se trata de edificios con segregación de funciones. El lobby de la planta baja y la zona de locutorios tienen características similares a los del patio de operaciones de un banco, las salas de operadoras se asemejan a talleres, las oficinas no difieren de las de una compañía de seguros y las plantas de equipamiento son de concepción y estética industrial.

Además de los cambios impuestos por la automatización los responsables de AT\&T comprendieron que la financiación necesaria para construir el servicio uni- 


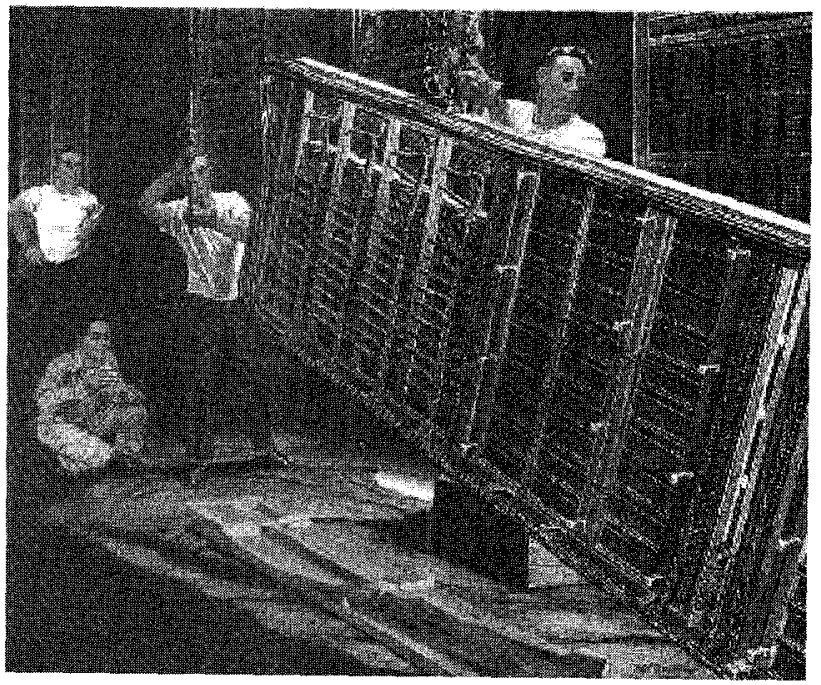

Fig. 4. Cuadrilla montando una central automática en un anuncio de Western Electric. Debido a la escasez de espacio esta labor era llevada a cabo manualmente por personal muy especializado. Fuente: Bell System Memorial.

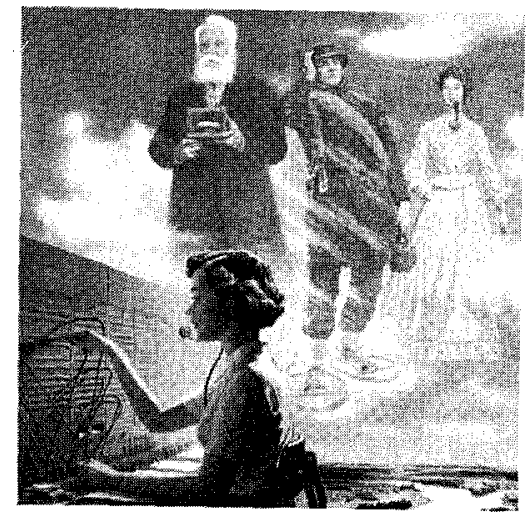

The Spirit of Sorvice That Kaept on Growing

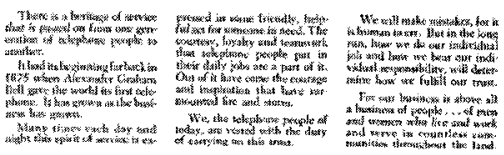

*xi .

Fig. 5. Anuncio de AT\&T de 1932 con las imágenes de Bell, la operadora y el técnico de servicio. Fuente: Bell System Memorial. 
versal, sólo podía lograrse aumentando la base de inversores. A partir de 1920, la compañia gastó grandes sumas en publicidad y la imagen pública se cuidó con esmero para atraer clientes e inversores. El derroche de lujo en las zonas abiertas al público de los edificios principales y las visitas organizadas formaban parte del programa publicitario. Las centrales adquirieron así, un valor de representación de la compañía, y esto favoreció las consideraciones estéticas en su diseño.

La publicidad contribuyó a crear una iconografía fácilmente reconocible por el público, que se utilizó como motivo decorativo en las centrales de nueva construcción. La figura paternal del anciano Graham Bell, la operadora siempre sonriente y el sufrido operario subido a un poste son los elementos más repetidos de un lenguaje visual adoptado por todas las compañías telefónicas del mundo.

\section{EL EDIFICIO BARCLAY-VESEY}

En 1921, un joven arquitecto, Ralph Thomas Walker, del estudio McKenzie, Voorhees \& Gmelin recibió el encargo de proyectar la sede principal de la New York Telephone Company, la filial local de AT\&T. La elección del estudio no era casual, puesto que el fundador Cyrus Eidlitz ${ }^{4}$ fue el autor de la primera central de la compañía ${ }^{5}$ en 1885 y desde entonces existía una estrecha relación profesional entre la firma y la NYTC.

El edificio, que habría de construirse en el Lower Manhattan, entre las calles Barclay y Vesey que le dan nombre, estaba destinado a reunir seis mil empleados hasta entonces dispersos en diecisiete sedes, más equipo de conmutación automático. Un encargo de esta envergadura debería haber sido dirigido por Paul Gmelin, el arquitecto jefe. Sin embargo, fue Walker el escogido. Tenía entonces 30 años y no había aún realizado ninguna obra de grandes dimensiones. A diferencia de lo que sucedía en Europa, el oficio de arquitecto en Estados Unidos no exigía una titulación específica en la materia. Walker se había formado como meritorio en un estudio de Providence, su ciudad natal, y posteriormente en el MIT. En esta prestigiosa universidad había destacado por su talento pero la abandonó tras un enfrentamiento con el decano. Posteriormente, la Gran Guerra le mantuvo alejado de la práctica arquitectónica, de manera que puede decirse que la New York Telephone Company había confiado su edificio principal a un inexperto. Esta circunstancia se repitió casi exactamente cuatro años después en España cuando

${ }^{4}$ Cyrus era hijo de Leopold Eidlitz (1823-1908), uno de los primeros arquitectos formados en el continente europeo que llevaron a mediados del siglo Xix una visión de la arquitectura diferente del modelo británico, único referente hasta entonces de los profesionales de la joven nación. Véase HaNDLIN, David P.: «American Architecture», Thames \& Hudson, Londres, 1989. pág. 103.

5 ARMS, M.: Op. cit., pág. 69. 
ITT encomendó el proyecto emblemático de Gran Vía a Ignacio de Cárdenas, un recién titulado. Esta coincidencia creemos que no es circunstancial. La elección de un arquitecto brillante pero con poca experiencia era la opción más acertada si lo que la compañía pretendía era tener control del resultado final.

La calidad de Walker como arquitecto y su proyección dentro de la compañía habían quedado acreditadas por su participación en el concurso internacional convocado en 1922 por el Chicago Tribune. El diario de la metrópolis de los Grandes Lagos que se anunciaba como el más importante del mundo, deseaba contar con una nueva sede digna de esa posición. El premio de 100.000 dólares atrajo a los mejores profesionales del país y a los arquitectos europeos más innovadores.

El rascacielos de oficinas era un tipo de edificio familiar para los arquitectos norteamericanos, pero exótico para los europeos. Las propuestas de los primeros se acogieron por regia general al rascacielos-columna tipo de estilo neogótico, mientras que las propuestas europeas rozaron en algún caso lo sorprendente. El concurso sirvió para que la arquitectura de vanguardia europea abordara casi por primera vez el diseño de edificios de esta naturaleza. El ganador fue el de Hood y Howells, en estilo neogótico, muy adecuado al gusto del comitente. Walker presentó un proyecto en la misma línea, en el que demostraba su talento práctico, pero sin salirse de lo establecido. El concurso tuvo un impacto indeleble en la arquitectura de los años 20 gracias al diseño de Eliel Saarinen que quedó segundo. Pese a que el edificio debía construirse en Chicago, el arquitecto finlandés hizo una propuesta basada en la normativa neoyorquina conocida como Zoning Law de 1916, dando lugar a la característica disposición de cuerpos retranqueados en la cúspide del edificio que da personalidad a los rascacielos de los años 20 y 30 .

Del impacto del diseño de Saarinen da idea que no sólo Walker, sino el propio Hood en su Radiator Building de 1924, vieron su estilo profundamente modificado como consecuencia de este concurso.

La Zoning Law estaba destinada a evitar que el excesivo crecimiento en altura dificultase la iluminación y ventilación de las calles de Nueva York. La norma establecía, en función de la anchura de la vía, la altura máxima a partir de la cual se debía retranquear la construcción.

El proyecto definitivo para el Barclay-Vesey, de 1923, saca un máximo partido de las posibilidades expresivas de la composición mediante cuerpos retranqueados. El aspecto más llamativo del edificio, cuya construcción se llevó a cabo entre mayo de 1923 y febrero de 1926, es su rotundidad. La base ocupa todo el solar de forma trapezoidal y se eleva diez plantas. Sobre ella se eleva otro cuerpo con forma de $\mathrm{H}$ y el edificio se corona con una torre rotada 45 grados para alinearse con los grandes edificios de Broadway, en particular con el Woolworth Building, su ve- 


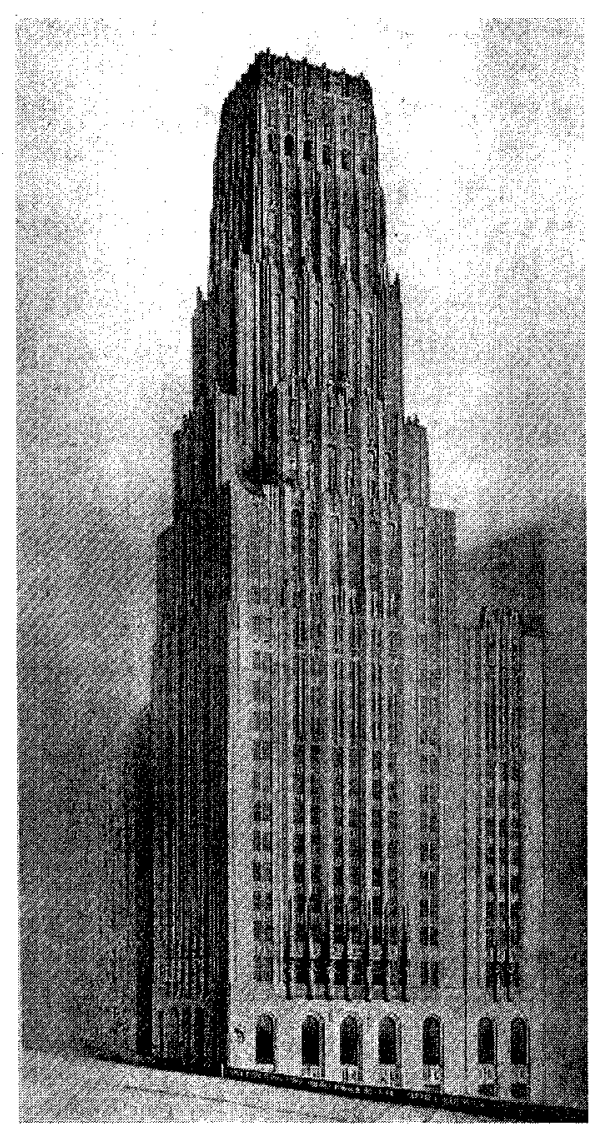

Fig. 6. Diseño de Eliel Saarinen para el concurso del Chicago Tribune.

cino visual. Sus 32 plantas y 152 metros de altura destacaban por su privilegiada posición en línea de costa en el skyline neoyorquino.

La decoración es una de sus marcas distintivas y creó un lenguaje que sería posteriormente seguido en numerosas centrales de todo el mundo. Walker pudo dar rienda suelta a su imaginación en este propósito no confesado de épater le bourgueois de su cliente. Desechó los adornos góticos y se decantó por una decoración con motivos vegetales tanto en las fachadas como en el lobby, muy influida por las ideas de Louis Sullivan y de la versión americana del movimiento Arts \& Crafts. Uno de los elementos más efectistas del edificio son los soportales con arcos de medio punto, en la calle Vesey, con figuras de leones, conejos y ardillas. Las dos entradas principales del edificio se encuentran enmarcadas por figuras de 


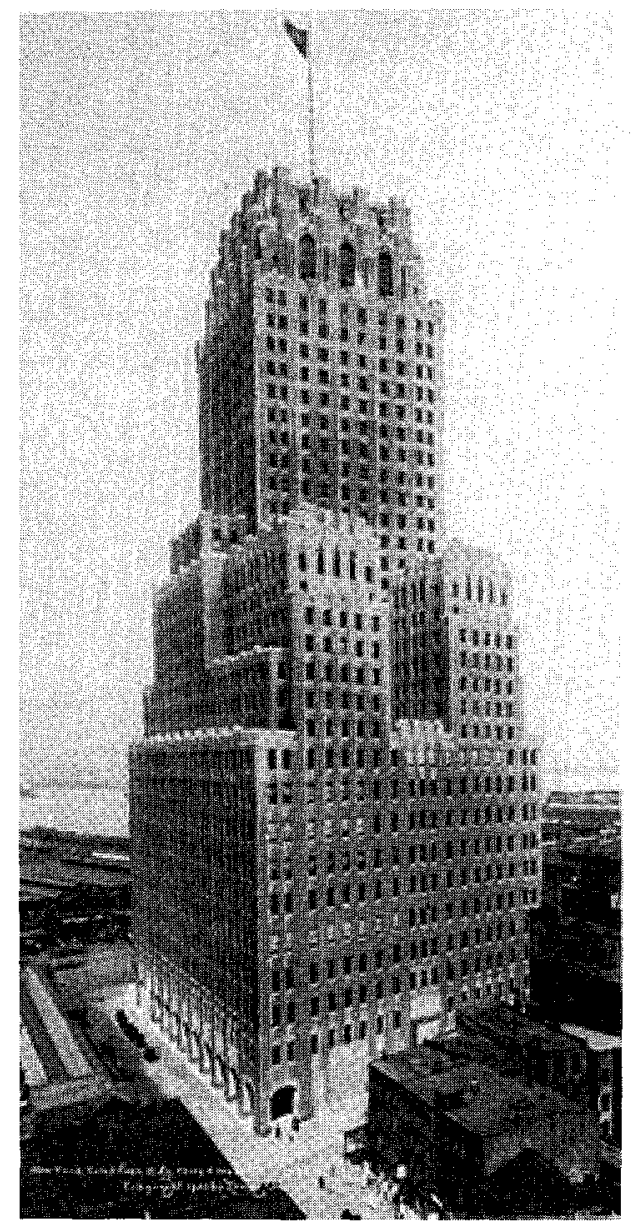

Fig. 7. Edificio Barclay-Vesey en una fotografía de Berenice Abbott, cuando destacaba en la orilla del East River. Fuente: University of Virginia.

niños tocando la flauta, un indio, osos, pájaros o racimos de uva, en una evocación de los abigarrados pórticos de las catedrales francesas que había conocido durante su servicio en la Primera Guerra Mundial. En el lobby, diez frescos, obra de Mack, Jenney y Tyler, decoran el techo ilustrando la historia de las comunicaciones. En el suelo se situaron dos medallones de bronce con los iconos más repetidos por todas las compañías telefónicas del mundo, la operadora y el técnico subido a un poste.

La decoración del edificio Barclay-Vesey es original, no por sus elementos, una acumulación de estilos diversos y hasta contradictorios, sino por ser el primer in- 


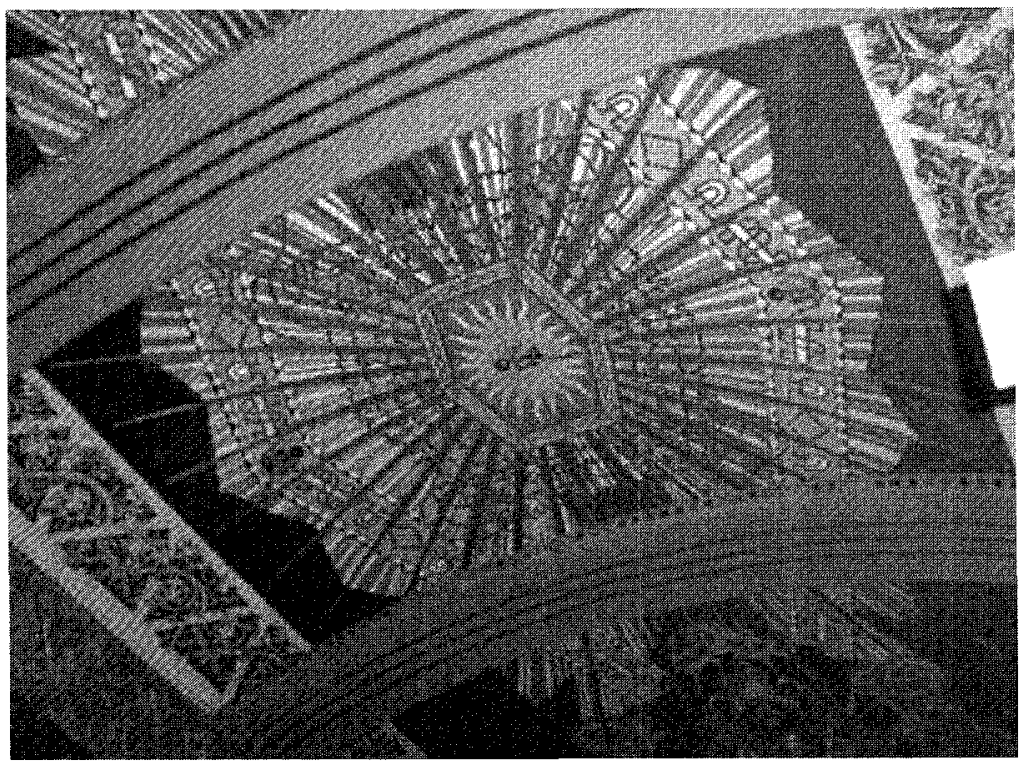

Fig. 8. Detalle de los frescos. Un teléfono se sitúa en el centro de esta alegoría solar. Curioso uso de símbolos de esquemas eléctricos (lámparas, relés y puentes de Wheatstone) en la cenefa decorativa. Fuente: Verizon.

tento de crear un programa iconográfico propio del negocio al que servía. En la actualidad, el edificio se valora como parte de la historia de la ciudad de Nueva York y Verizon ha invertido una cantidad elevada en restaurar los bajorrelieves de la entrada este y de buena parte de la fachada correspondiente, destruidos por el derrumbe del World Trade Center.

En manos de otro arquitecto menos creativo, es posible que el edificio BarclayVesey hubiese sido igual de funcional pero no muy diferente de los almacenes que to rodeaban. Aunque otras obras posteriores como el Empire State, el Chrysler Building o el Irving Trust del propio Walker han eclipsado la fama popular de este rascacielos, en el momento de su terminación causó una honda impresión. En 1927 recibió la medalla de oro de la Architectural League of New York por ser un símbolo de la nueva era industrial. Joseph Pennell, célebre paisajista del Lower Manhattan lo calificó como el edificio moderno más impresionante del mundo y ya hemos citado anteriormente el aprecio que sintió por él un arquitecto de ideas tan diferentes a las de Walker como Le Corbusier. 


\section{LA REGIÓN DE LOS GRANDES LAGOS}

El teléfono nació en Nueva Inglaterra. La región industrial de los Grandes Lagos fue el objetivo principal de expansión una vez que la primitiva compañía afianzó su negocio en la franja comprendida entre Washington y Boston.

De todas las grandes centrales de esta zona, la que más directamente recibió ef influjo de Walker, fue la de Cleveland. Este edificio, construido entre 1925 y 1927, acusa de manera singular el impacto que causó entre los profesionales el edificio Barclay-Vesey. Fue un encargo de la Ohio Bell Telephone Company al estudio Hubbell \& Benes, radicado en la misma Cleveland.

Benjamin Hubbell y Dominick Benes proyectaron una de las centrales más hermosas de la década, que hoy día sigue sirviendo al propósito con que se encargó. El edificio tiene 20 plantas, con unos poderosos piers ${ }^{6}$ decorativos y un inteligente uso de los retranqueos, que en esta ciudad no respondían a la normativa de construcción. El lenguaje creado por Saarinen y seguido por Walker se había adoptado ya como una posibilidad expresiva.

Al igual que otras centrales en todo el planeta, recibió el sobrenombre de Templo de la telefonía, un apelativo que se da en otras construcciones industriales. Las grandes empresas no tuvieron reparos en utilizar este tipo de denominaciones grandilocuentes para referirse a sus sedes centrales, con independencia de su ramo de actividad.

En Chicago, la cuna del rascacielos, no se construyó una central de calidad comparable a Nueva York o Cleveland. La razón es posible que esté en el estudio de cabecera de la Illinois Bell, el todopoderoso Holabird \& Roche.

Desde principios de siglo, la compañía telefónica y Holabird \& Roche establecieron una duradera relación profesional. La capacidad de trabajo de un gabinete tan importante era ideal para abordar varios proyectos en paralelo y cumplir los plazos con los que el cliente urgía. La contrapartida a esta eficiencia fue la falta de originalidad, pues las centrales telefónicas no eran el producto estrella de la firma, al contrario que en el caso de McKenzie, Voorhees \& Gmelin. Los arquitectos principales estaban concentrados en otros proyectos como el Chicago Board of Trade o el edificio Palmolive. En palabras de John August Holabird, hijo de John Archibald y también arquitecto: "We did an awful lot of telephone building in those days and it continued in the 1920 s... At one time there must have been twenty or thirty buildings each year's?

6 Pier es el término inglés para columna o pilar. En los edificios art-déco, se utiliza para designar específicamente las amplias líneas de ladrillo o piedra que forman la retícula de la fachada.

7 HoLABIRD, John August: Transcripción de sus memorias habladas dentro del proyecto «Oral History of Chicago Architects". 


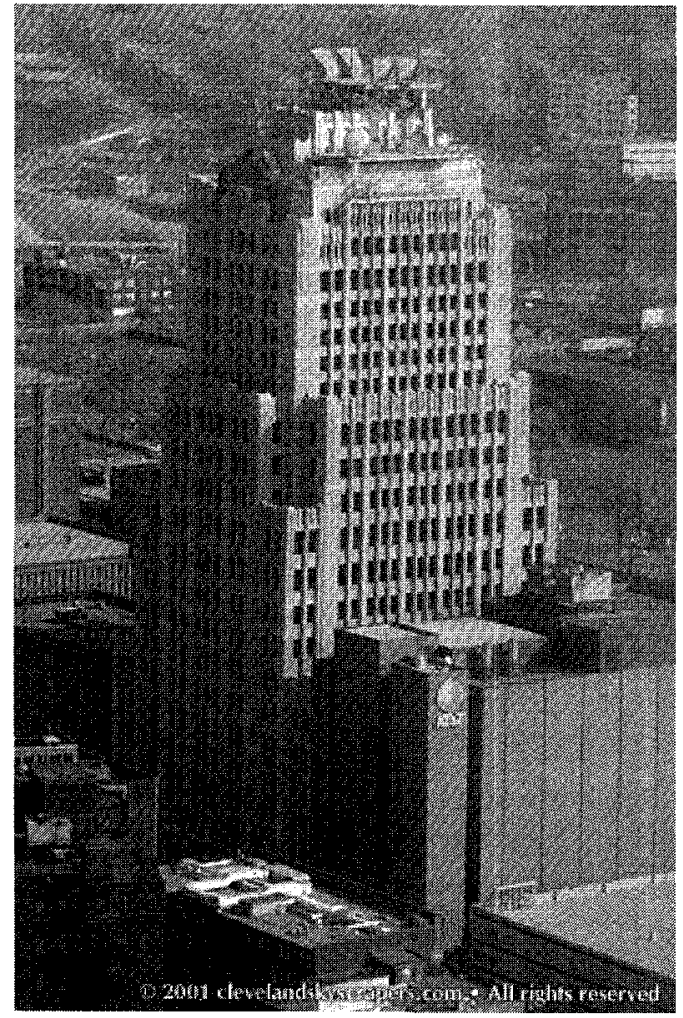

Fig. 9. Edificio Ohio Bell. A la derecha puede observarse un edificio anejo de los años 70 , para ampliar el espacio disponible. Fuente: Ohio Skyscrapers.

La ausencia de innovación en este periodo se revela en la continuidad de la decoración neogótica en los edificios, y el mantenimiento del esquema de rascacielos-columna.

En el estado de Michigan, la compañia local, Michigan Bell Telephone trabajó muy estrechamente desde principios de siglo con Smith, Hinchman \& Grylls. Más que un estudio de arquitectura, SH\&G era (y sigue siendo) una gran empresa de ingeniería de proyectos. Entre 1920 y 1940 construyó numerosas centrales por todo el estado.

La central de mayor calidad arquitectónica no es la principal de Detroit sino una de tamaño mediano denominada Columbia. Se construyó en 1927, para albergar 7000 líneas automáticas. SH\&G utilizó en este caso el estilo art-déco. La ciudad de Detroit es una metrópolis industrial, muy extensa y poco articulada. Los edificios de 


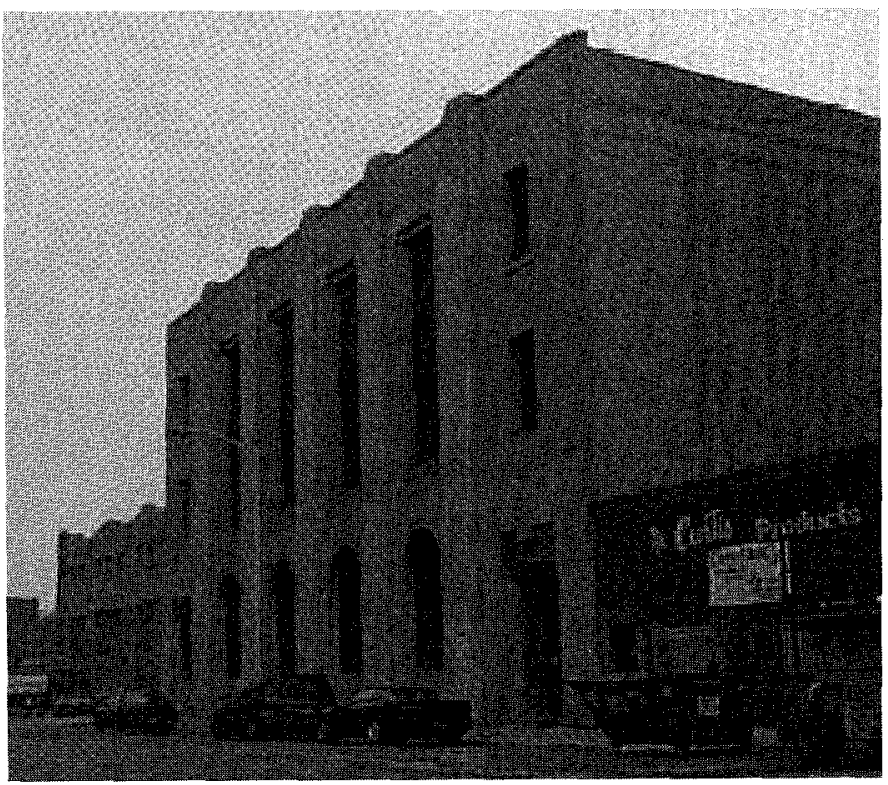

Fig. 10. Central Detroit-Columbia. Fuente: City of Detroit Planning.

la compañía telefónica se distribuyeron según criterios de pura eficacia geográfica. Algunos de ellos son de estética muy discutible y otros, situados en distritos conflictivos están rodeados de alambradas. El mérito de SH\&G no estuvo en la relevancia arquitectónica de los edificios, sino en la capacidad para satisfacer las necesidades de su cliente. De los tres grandes constructores de centrales de la década de los 20, fue este estudio el que mejor supo comprender la esencia del negocio telefónico y no es casualidad que sea el único que lleva trabajando ininterrumpidamente en este sector desde hace un siglo.

\section{CENTRALES DEL OESTE}

El Oeste fue incorporado a Estados Unidos gracias a los avances de las comunicaciones, en especial el ferrocarril y el telégrafo. Esta sensación de alejamiento de los centros de poder político y económico de la Costa Este era aun palpable en 1920, cuando la aviación comercial no era más que un negocio embrionario.

Hay que tener presente que la arquitectura de las ciudades de frontera es muy distinta de las de la Costa Este. Las normas urbanisticas eran mucho más laxas, y 

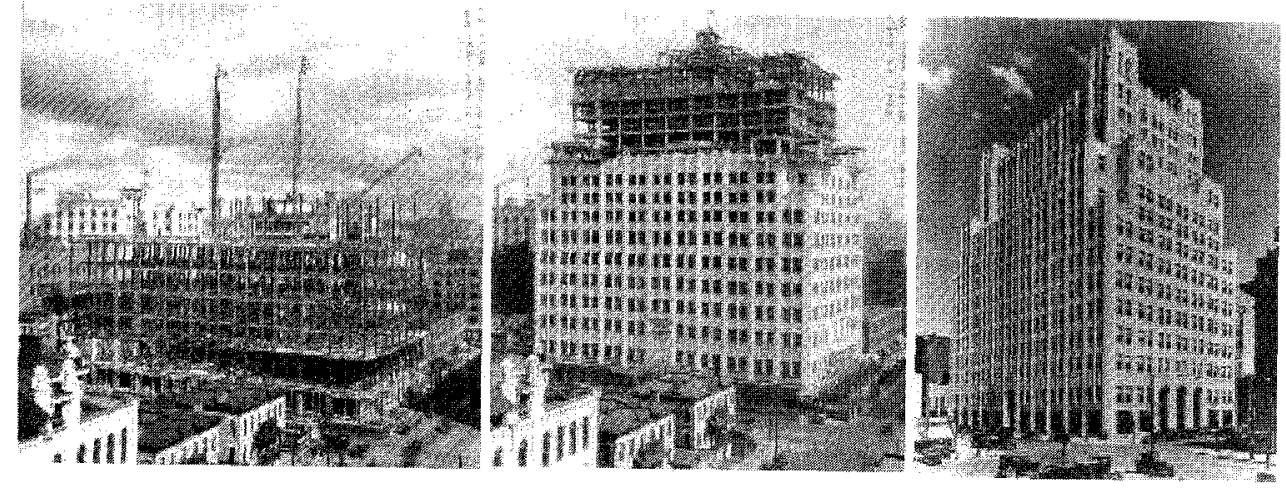

Fig. 11. Proceso constructivo de la central de Denver.

Fuente: The Telecommunications History Group.

no existía un problema de salud pública como el que llevó a establecer la Zoning Law. Por este motivo, las centrales del Oeste suelen estar en manzanas exentas y las compañías no buscaban ubicarlas en el downtown comercial, ya que este concepto prácticamente carece de sentido en las aglomeraciones urbanas de la región.

La puerta tradicional del Oeste es St. Louis y en esta ciudad se levantó uno de los edificios telefónicos más espectaculares. El conocido como Grand Exchange (1925) es obra del estudio Mauran, Rusell \& Crowe. Con 28 plantas, su figura está entre el castillo almenado y el zigurat. Aunque contiene elementos art-déco, su aspecto de fortaleza, que se aprecia mejor en la postal de época, y la mezcla de estilos lo convierten en uno de las centrales con más personalidad de la década.

Otra central-fortaleza es la de Denver, finalizada en 1929, obra de William Bowman por encargo de la Mountain-States Telephone Company. Se conoce como el último palacio del sistema Bell, pues inmediatamente después llegó la Gran Depresión y acabó el periodo de esplendor constructivo.

De finales de la década, aunque terminada en 1932, es la central de Minneapolis, de Rhodes Robertson del estudio Hewitt \& Brown. Es un rascacielos art-déco de factura muy elegante y detalles decorativos exquisitos. Aunque en las fotografías no puede apreciarse con detalle, la verticalidad rotunda de los piers es suavizada por las láminas de aluminio grabado que confieren detalles azulados a la fachada. Robertson tomó como motivo principal de su programa la electricidad y emplea los rayos como recurso decorativo.

En California, sobresale por sus dimensiones y belleza la sede central de Pacific Bell, en San Francisco. El rascacielos de 26 plantas se construyó en 18 me- 


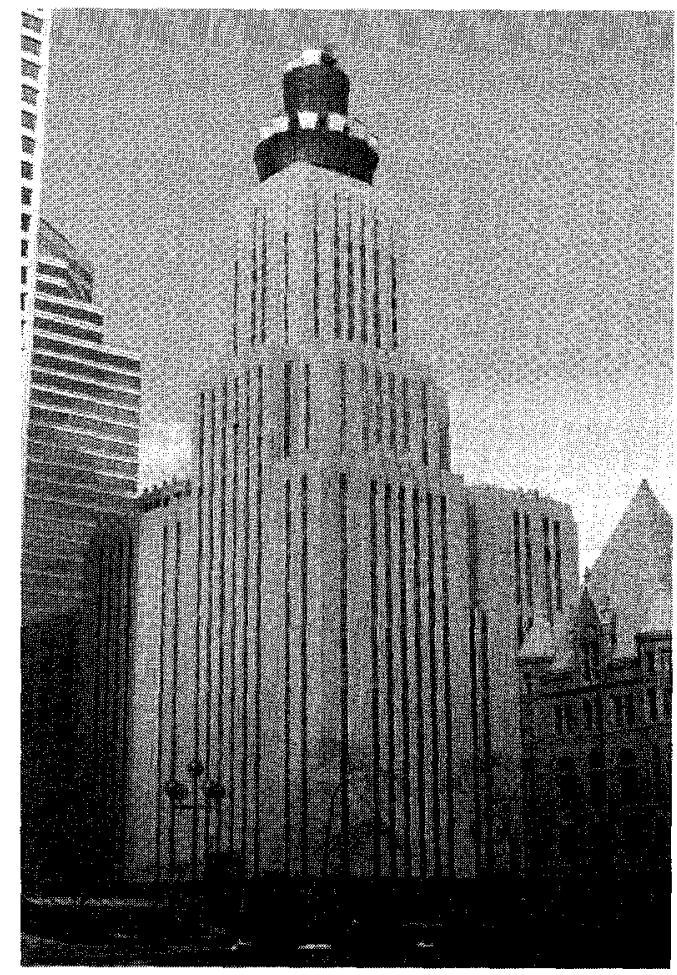

Fig. 12. Central de Minneapolis, imagen actual.

ses, entre 1924 y 1925 y supuso la irrupción de la arquitectura art-déco de grandes dimesiones en Californa. El arquitecto fue A. Cantin, que trabajaba para el estudio Miller \& Plueger. Su inauguración tuvo un gran valor simbólico pues se erigió sobre el solar de una antigua central destruida por el gran terremoto de 1906.

\section{CONSERVACIÓN DE LOS EDIFICIOS}

En nuestros días, se asiste a un proceso de apreciación de la calidad arquitectónica de las centrales. Este fenómeno se manifiesta con fuerza en Estados Unidos donde algunos de los edificios objeto de este estudio están recibiendo protección ya sea municipal, estatal o federal. El desarrollo urbano norteamericano se ha llevado, literalmente, por delante buena parte de sus edificios emblemáticos. En un país con tan escaso pasado algunas centrales tienen un valor como referente simbólico que resulta difícil comprender en el Viejo Continente. 

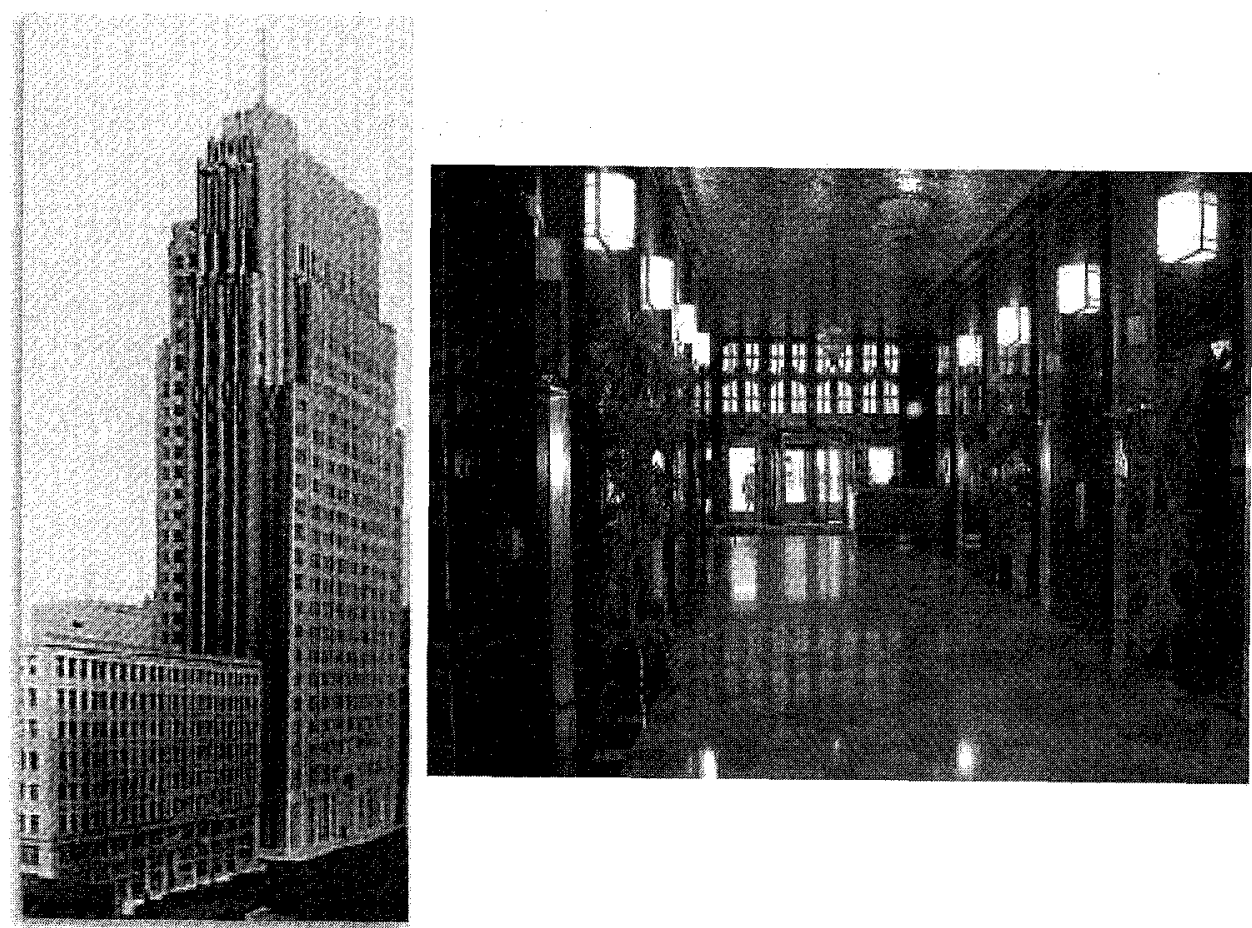

Fig. 13: Editicio Pacific Bell, exterior y detalle del lujoso lobby. Fuente: Museum City of San Francisco.

Como es bien sabido, la mejor forma de proteger un edificio es que continúe sirviendo para el propósito con que se construyó. Ese es el caso de la mayor parte de las centrales, por las razones técnicas y económicas que hemos expuesto. Las compañías propietarias tienen incentivos de tipo fiscal y de mantenimiento de una buena imagen pública para invertir fuertes sumas en sus centrales emblemáticas. No sucede así con otras situadas en zonas urbanas menos privilegiadas o de construcción más reciente.

\section{EL MODELO AMERICANO LLEGA A ESPAÑA}

La historia de la telefonía en España tiene un momento clave, la firma en agosto de 1924 de un contrato entre el estado y la International Telephone and Telegraph por el que se concedía a esta empresa «la organización, reforma y ampliación del servicio telefónico español». En la práctica, equivalía a ceder la explotación en régimen de monopolio a cambio de un ambicioso plan de inversiones. 
International Telephone and Telegraph ${ }^{8}$ impuso desde el principio los métodos empresariales americanos. La Compañía Telefónica Nacional de España se constituyó a imagen de una operadora de aquel país, con los puestos claves controlados por directivos e ingenieros de ITT en los primeros años de actividad. La operación se puede calificar de éxito pues en pocos años se logró alcanzar la cifra de 300.000 abonados. La clave de este crecimiento fue un ambicioso programa de instalaciones tanto de equipos automáticos como de líneas interurbanas e internacionales.

La responsabilidad de la edificación de nuevas centrales recaía en un departamento al frente del cual se colocó a Ignacio Cárdenas, un arquitecto completamente inexperto pues había obtenido el título en $1924^{\circ}$. Es la misma situación que hemos visto en el caso de Walker, sólo que en esta caso la intención de control era aún más evidente pues se trataba de un empleado directo de la empresa. Aunque en principio se pensó en sacar a concurso público los edificios de Madrid, BarceIona y Sevilla, sólo se cumplió en el caso del segundo. Debido a los problemas aparecidos en este consurso, ITT decidió que la dirección de todo el programa la haría directamente Cárdenas y así en 1925 se encontró con el encargo de proyectar el edificio emblemático de Gran Vía.

El control de ITT se ejercía in situ por el ingeniero Aldrich Durant y su grupo de profesionales norteamericanos. Durant permaneció en España hasta finales de 1927, momento en el cual se consideró que el departamento de Cárdenas estaba ya capacitado. El grupo de Durant se iba trasladando de un país a otro a medida que la ITT extendía su presencia.

Una buena prueba de esta influencia es que todo el cálculo de estructuras se hizo de acuerdo con el reglamento de edificación de Nueva York y que en buena parte de los planos de la estructura aparecen unidades de medida anglosajonas. Los perfiles fueron contratados a fundiciones españolas, y la inexistencia de algunos tipos comunes en Estados Unidos obligó a hacer ciertas modificaciones ${ }^{10}$.

Nos interesa es destacar como el modelo de trabajo americano se adoptó por Telefónica en el amplio programa de construcciones de los primeros años de

${ }^{8}$ La historia de ITT está muy ligada a la figura de su fundador Sosthenes Behn, ex-militar y aventurero. El comercio de azúcar le llevó a obtener una licencia telefónica en 1920 en las Islas Vírgenes. De ahí pasó a Puerto Rico y Cuba. Su expansión internacional continuó por España, México, Rumanía y la mayor parte de América del Sur. Con su facilidad para moverse en los círculos de poder, obtuvo concesiones de la mayor parte de los presidentes de repúblicas latinoamericanas y fue uno de los pocos empresarios extranjeros que entró en el círculo más próximo a Adolf Hitler.

9 Véase Navascués, Fernández, Musoles y romeo: El edificio de la Telefónica. Espasa Calpe, Madrid, 1984. Es un manual de referencia obligada para conocer la gestación y desarrollo del proyecto del edificio de Gran Vía.

${ }^{10}$ Musoles, Fernando: La estructura del edificio de la Telefónica: Aspectos constructivos», en NAVASCUÉs, et alii, págs. 179-204. 
actividad. Sabemos que los directivos de ITT sugirieron a Cárdenas que visitase en Nueva York a Louis S. Weeks, arquitecto jefe:

«Pero como los americanos estaban en la idea de que en España estábamos atrasadísimos en todo lo relacionado con la arquitectura moderna encontraron la solución a mi supuesta ignorancia enviándome a Nueva York donde el arquitecto de la ITT me orientaría sobre ello. Aclaro que esto yo lo consideré normal pues mis pocos años tenían que inspirar poca confianza» ${ }^{11}$.

Esta visita se produjo a finales de 1925, cuando estaba en proceso de construcción el edificio Barclay-Vesey. Cárdenas acudió con Weeks a visitar varias obras en curso, e imaginamos que una de ellas debió ser la del edificio de Walker, aunque no nos consta. De los rascacielos neoyorquinos, el arquitecto madrileño se había quedado con el dato de que el estilo gótico había pasado de moda y que se usaba el retranqueo (cita el término set-back en inglés) como forma de articulación.

De Louis $\mathrm{S}$. Weeks la información disponible es muy escasa. Había estudiado en la École des Beaux-Arts de París, y se le atribuyen algunos de los edificios de las filiales de ITT como el de Bucarest (1933), junto con su compatriota Walter Froy y Van Saanen Algi. Puede que este dato no sea muy exacto dado que también hemos encontrado citas que erróneamente le asignan la paternidad de la central de Gran Vía. Weeks actuaba más como un ejecutivo de alto nivel de la compañía que se aseguraba del cumplimiento de los plazos y requisitos impuestos por ITT y dejaba la confección de los proyectos en manos de los que eran sus subordinados, Cárdenas en Madrid y Van Saanen en Bucarest ${ }^{12}$.

Para dar una idea del ritmo de trabajo, baste decir que en 1927 estaban en marcha las obras de 27 centrales. Este ritmo sólo puede sostenerse si todo el proceso se planifica y gestiona con criterios industriales, para lo cual es preciso que haya una organización sólida y financieramente poderosa.

11 Memorias manuscritas de Ignacio Cárdenas, reproducción facsímil en NAVASCuÉs, et alii.

12 En este fragmento de la historia de la compañía que puede leerse en la página web de Romtelecom (http://www.romtelecom.ro), vemos que el proyecto de Bucarest siguió los mismos pasos que los de las centrales de la filial española. «The first initiative of building a Telephone Palace in Bucharest was taken in 1930. On August 25, 1931, the Romanian Telephone Company PIC. (RTC) obtained the authorisation to build a Telephone Palace, on its property located at 35, Victoriei Road. The architect Edmond Van Sanen Algi designed the architectural plan of the edifice.

Having decided to start this project as soon as possible, at the end of 1931 RTC signed an Agreement with "Union", a Civil Engineering Limited Company, whose object resided in casting the foundation of the building. The Palace metal skeleton frame was produced by the Steel Works in Resita. The Agreement signed with the Resita Works did not only provide for the steel quality, but also for the manufacturing procedures, as well as for girder riveting and placement. The acceptance of works was made in 1933. Finishing works included installation of heating, ventilating, water supply utilities, elevators and telecommunications equipment». 


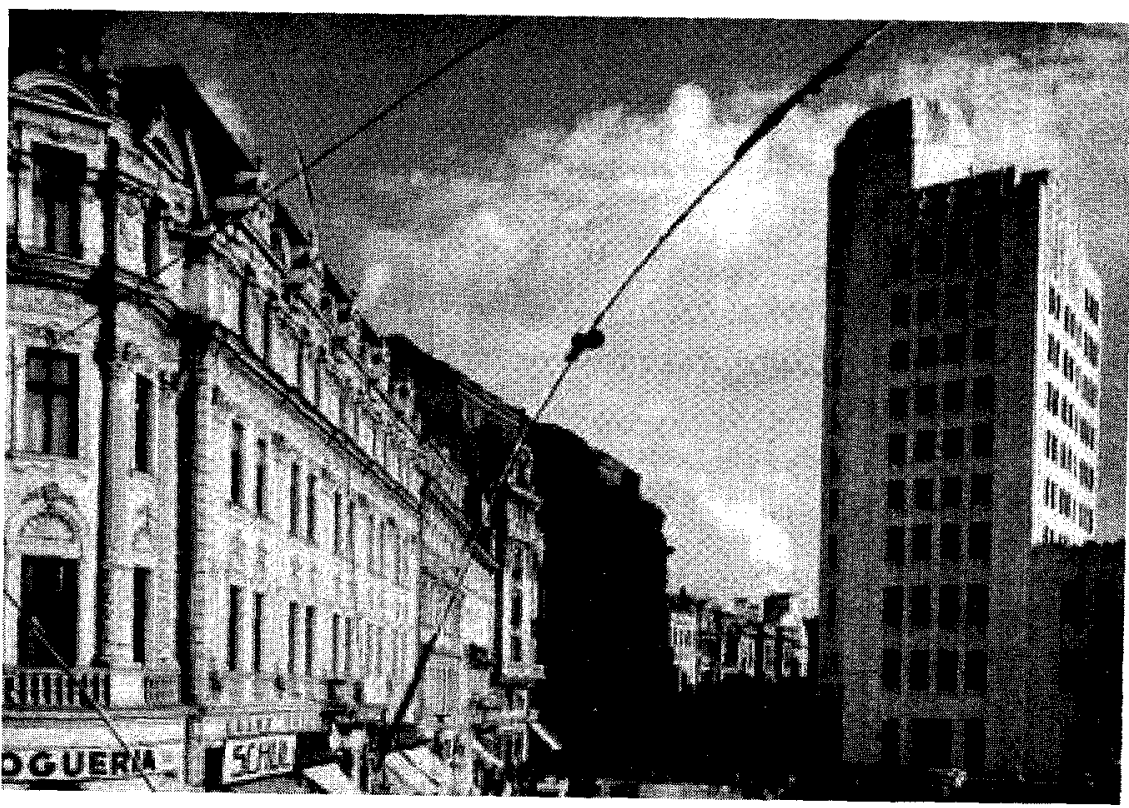

Fig. 14. Edificio /TT de Bucarest antes de la I/ Guerra Mundial (a la derecha de la imagen). Fuente: Romtelecom.

Las memorias manuscritas de Ignacio de Cárdenas revelan la adopción de la ideología empresarial y el modo de trabajo americano.

En unas pocas líneas, Cárdenas resume la doctrina que se había impuesto en Estados Unidos:

«En la enorme labor que la Compañía realiza para dotar a España de un servicio telefónico modelo, es el edificio un factor importantísimo para la garantía del éxito que todos perseguimos.

Difícil comparación tiene el edificio telefónico con otros destinados a fines parecidos, pues si es esencialmente un edificio de carácter industrial, es también una embajada de la Compañia en las ciudades españolas, y ha de ser, como ella, popular, suntuoso, útil y rico. También es un anuncio. Sin el anuncio fracasan hoy en día todas las empresas que del público viven, y un buen anuncio ha de estar enclavado en el mejor lugar de la ciudad. Pero de nada serviría que estuviese inmejorablemente situado, en lo que a circulación y vida ciudadana se refiere, si su situación obligase a una instalación difícil o costosa de las líneas urbanas e interurbanas».

El programa arquitectónico debe supeditarse a la utilidad. En relación con las complicadas tareas de montaje de los equipos que hemos puesto de relieve en el capítulo segundo, Cárdenas escribió: 
«Las plantas o distribución interior son la parte más importante del proyecto, y esta distribución, en aquellos de nuestros edificios que han de alojar un equipo automático, está supeditada a que éste se monte en las mejores condiciones, sacrificando gustosos a menudo un mayor efecto decorativo, por ejemplo, en una escalera, e incluso obligando a modificar la fachada. Se piensa siempre en el porvenir, y en los cálculos de resistencia se prevé la posibilidad de añadir nuevos pisos o variar la distribución primera».

El arquitecto menciona también otros elementos claves del modelo americano, como la visita concebida como instrumento publicitario y la apariencia externa a las peculiaridades de cada región.

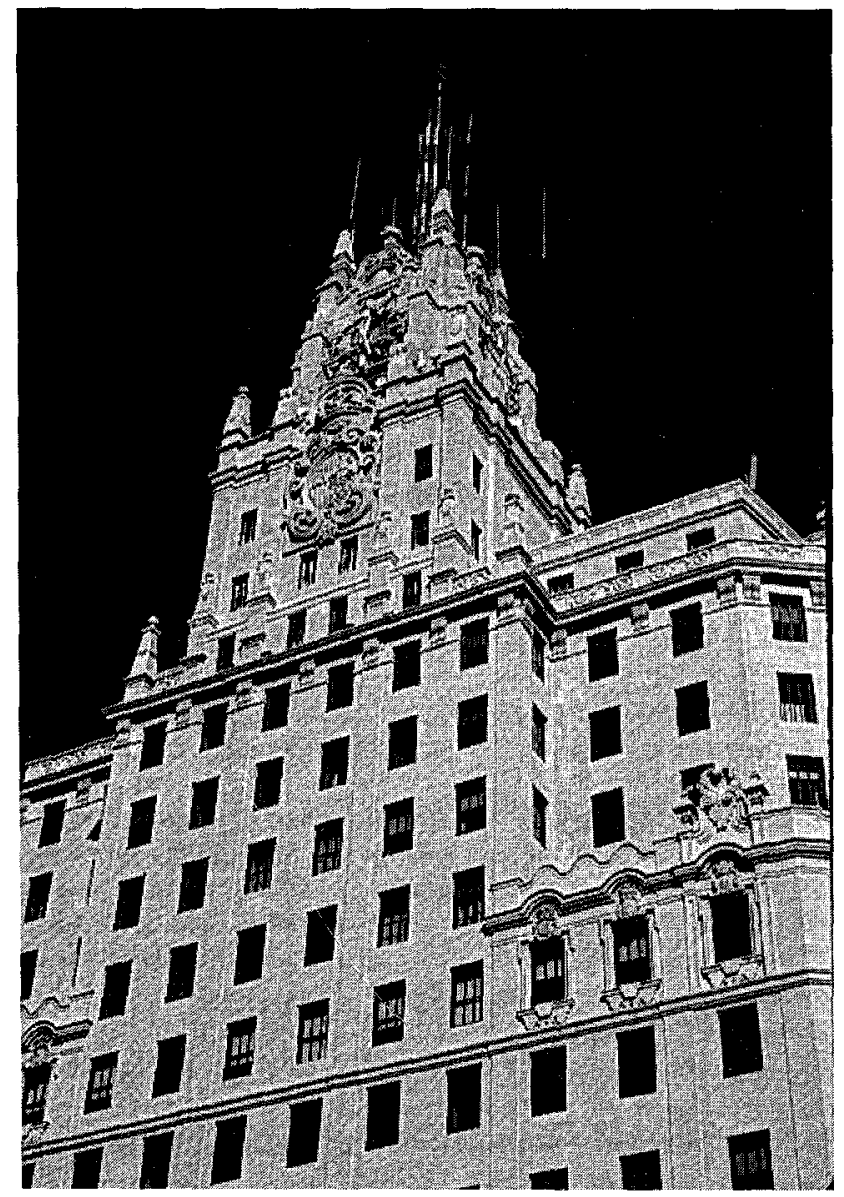

Fig. 15. Edificio de Gran Vía. Fuente: Telefónica. 
"La idea nacional de nuestra Compañía se afirmará en las fachadas de sus edificios, los cuales pretendemos siempre que armonicen con el carácter peculiar de cada población, y así se levantó en Santander la primera central de un marcado estilo montañés. Los edificios de Barcelona, Zaragoza y Bilbao son sobrios, clásicos y fuertes. Alegres y luminosos, el de Valencia y la sucursal de El Grao. En el de Sevilla se empleará toda la riqueza decorativa del arte antiguo y moderno sevillano. En las Arenas, en Vizcaya, haremos una Central que se asemejará a un pintoresco caserío vasco, y el de la Gran Vía, de Madrid, imponente, fuerte, majestuoso y muy español y madrileño, edificio que será el cerebro y el corazón de la vasta organización en que trabajamos".

El programa decorativo seguía siendo el de la arquitectura regionalista y ecléctica española de principios del siglo Xx. La construcción de los primeros edificios de la Telefónica coincidió en el tiempo con la llegada a España de las ideas de la arquitectura moderna, pero todos ellos fueron diseñados en clave regionalista.

ITT no habría aceptado con facilidad lo contrario, las memorias de Cárdenas nos cuentan su cordial enfrentamiento con Weeks, empeñado en que la fachada de Gran Vía fuese una copia de la Casa de las Conchas salmantina. La solución adoptada, siguiendo el criterio del arquitecto español, de remitirse al barroco madrileño, resultó mucho más adecuada a la estética de la nueva avenida.

\section{BIBLIOGRAFÍA}

ARMS, Meredith: The architecture of Ralph Thomas Walker: 1919-1930, Tesis doctoral, Rutgers University, 1995.

ÁlvARO MOYA, Adoración: Changing patterns of ownership and labor management under institutional regimes: The case of Telefónica in Spain, 1924-1970, Actas del EHBA 6th Congress, Helsinki, 2002

BEnSON, Reuel: The First Century of Telephone in Conneticut, University of Conneticut, 1978.

BoEtTInger, H. M.: The telephone book: Bell, Watson, Vail and American life, 1876-1983, Stearn, New York, 1983.

BRUEGMANN, Robert: "Collisions culturelles: projects européens et améircains pour le concours du Chicago Tribune de 1922", en Chicago, Naissance d'une métropole, catálogo de la exposición celebrada en el Musée d’Orsay, págs. 302-316, París, 1987.

CASSON, Herbert N.: The history of the telephone, Chicago, 1910. Edición electrónica en http://www.gutenberg.net.

HANDLIN, David P.: American Architecture, Thames \& Hudson, Londres, 1989.

HolABIRD, John August: Transcripción de sus memorias habladas dentro del proyecto "Oral History of Chicago Architects".

HUGHES, Jonathan: American economic history, Scott Foresman \& Co., New York, 1987

HuxTABLE, Ada Louise: El rascacielos. La búsqueda de un estilo, Nerea, Madrid, 1988.

NAvascués, Fernández, Musoles y Romeo: El edificio de la Telefónica, Espasa Calpe, Madrid, 1984.

PoITRAS, Claire: «Exploiter, vendre et consommer un nouveau service public. Le dévelopment du réseau de télephone a Montreal». Comunicación en el coloquio «El desarrollo urbano de Barcelona y Montréal en la época contemporánea". Universitat de Barcelona, 1997.

Romero Frías, Rafael: Colección histórico-tecnológica de Telefónica, Telefónica de España, Madrid, 1992.

WEIMAN, David F.: Building Universal Service in the Early Bell System, CUNY, 2003. 
